\title{
The sup-completion of a Dedekind complete vector lattice.
}

\author{
Youssef Azouzi and Youssef Nasri \\ Research Laboratory of Algebra, Topology, Arithmetic, and Order \\ Department of Mathematics \\ Faculty of Mathematical, Physical and Natural Sciences of Tunis \\ Tunis-El Manar University, 2092-El Manar, Tunisia
}

\begin{abstract}
Every Dedekind complete Riesz space $X$ has a unique sup-completion $X^{s}$, which is a Dedekind complete lattice cone. This paper aims to present a systematic study this cone by extending several known results to general setting, proving new results and, in particular, introducing for elements of $X^{s}$ finite and infinite parts. This enuables us to get a satisfactory abstract formulation of some classical results in the setting of Riesz spaces. We prove, in pareticular, a Riesz space version of Borel-Cantelli Lemma and present some applications to it.
\end{abstract}

\section{Introduction}

Completeness is a desirable property for anyone dealing with Riesz spaces. Such spaces may have several kinds of completeness. Under laterally completeness suprema exist for all disjoint families. Under Dedekind completeness suprema exist for all nonempty bounded subsets from above. Dedekind complete Riesz spaces are also called order complete; this can be explained by the fact that in those spaces order Cauchy nets are order convergent. If we consider uniform convergence instead of order convergence we talk about uniformly completeness. Recently, the notion of unbounded order convergence (uo-convergence) has received much attention (see for example [26, 13, 1, 15]) and then one can be interested in uo-complete Riesz spaces. Uo-completeness 
means that every uo-Cauchy net is uo-convergent. It is quite surprising that Uo-completeness is equivalent to universal completeness. This is the main result in [1, Theorem 17]. When nets are replaced by sequences we speak about sequential completeness. In general, sequential completeness is not equivalent to completeness. If the spaces we consider fail to be complete there is a way to make them complete by embedding those spaces in complete spaces (of course, under some additional assumptions). The notion of completion is then helpful and useful. Among several types of completion in Riesz space Theory we will focus in this paper on the notion of sup-completion. This notion has been introduced by Donner in [11]; it is perhaps less known and less frequently used in the literature. Several recent papers present important applications of it, essentially published by Grobler [14, 15], Grobler and Labushangne [17, 18, and the first author [1]. Sup-completion is used by Grobler in [14] to constructed Daniell integral in Riesz spaces. Recently the first author used this notion to prove the equivalence between universal completion and unbounded order completion. The current paper can be broken into two parts, both of them deal with sup-completion. The first part is devoted to the study of the sup-completion of a Dedekind complete Riesz space itself. It starts by a brief review of what is known and then presents several new results, namely the introduction of finite and infinite parts of elements of $X^{s}$. The second part is motivated by the famous Borel-Cantelli Lemma. It aims to give Riesz space generalizations of this lemma and provide some applications. The Borel-Cantelli Lemma contains two parts. Its first one is stated under the assumption that the series $\sum_{k} \mathbb{P}\left(A_{k}\right)$ is convergent, where $\left(A_{n}\right)$ is a sequence of events, and its generalization to the setting of Riesz spaces is quite obvious. This has already been done by Kuo, Labuschagne and Watson in [25]. Another generalization of the first Borel-Cantelli lemma, due to Barndorff-Nielsen and Balakrishnan-Stepanov, have been recently translated to the setting of Riesz spaces by Mushambi, Watson and Zinsou [27]. The second part of the Borel Cantelli Lemma, however, has not received enough attention in the frame of Riesz spaces setting and it is obvious that its generalization requires more care. The condition $\sum_{k} \mathbb{P}\left(A_{k}\right)=\infty$ forces us to work in the space $X^{s}$. A good understanding of that cone is then required in order to get satisfactory translation of several results from classical theory of probability to the setting of Riesz spaces. A series in a Riesz spaces may be converging on some band $B$ and diverging on its orthogonal $B^{d}$ and we can be interested in determining the largest band on which the series is con- 
verging. So, we are led to consider the finite and infinite parts of an element in $X^{s}$. This allows us to get more meaningful statements in the case of Riesz spaces.

An outline of the paper is as follows: Section 2 contains some preliminaries on several types of convergence considered in Riesz spaces. Section 3 is devoted to the notion of sup-completion: we will describe the basic properties of the cone $X^{s}$ and extend several of them. We introduce for an element $x$ in $X_{+}^{s}$ its finite and infinite parts and use these notions to extend BorelCantelli Lemma in Section 4. In this section we present a new version of the first Borel Cantelli Lemma (BCL1), which extends the one obtained earlier by Kuo, Labushagne and Watson and prove a Riesz space version of the second Borel-Cantelli Lemma (BCL2). The last section, Section 5, provides several applications to the previous sections and especially to Borel-Cantelli Lemma.

\section{Preliminaries}

Throughout $X$ denotes a Dedekind complete Riesz space. So, every band in $X$ is a a projection band. For a band $B$ we denote by $P_{B}$ the associated band projection. If $B=B_{x}$ is a principal band generated by $x$, we write $P_{x}$ instead of $P_{B_{x}}$. If $P$ is a band projection we let $P^{d}:=I-P$ denote the band projection on the band $B^{d}$. The universal completion of $X$ is denoted by $X^{u}$, while its sup-completion is denoted by $X^{s}$.

We will deal in this paper with three modes of convergence; each defines a linear convergence structure on $X$ in the sense of . We briefly recall their definitions and some basic and important facts about them that will be useful for us. The first one, and the most useful, is the order convergence.

Definition 1 We say that a net $\left(x_{\alpha}\right)_{\alpha \in A}$ in a vector lattice $X$ is order convergent to $x$ if there exists a 'dominating' net $\left(y_{\beta}\right)_{\beta \in B}$ satisfying $y_{\beta} \downarrow 0$ and for any $\beta \in B$ there exists $\alpha_{0} \in A$ such that $\left|x_{\alpha}-x\right| \leq y_{\beta}$ for every $\alpha \geq \alpha_{0}$. We write $x_{\alpha} \stackrel{o}{\longrightarrow} x$ or, more simply, $x_{\alpha} \longrightarrow x$.

The second mode of convergence can be viewed as an abstraction of almost surely convergence.

Definition 2 We say that a net $\left(x_{\alpha}\right)$ in a Riesz space $X$ unbounded order converges (or, uo-converges) to $x$, and we write $x_{\alpha} \stackrel{\text { uo }}{\longrightarrow} x$, if for every $u \in X_{+}$, the net $\left|x_{\alpha}-x\right| \wedge u$ is order convergent to 0 . 
If $X$ has a weak order unit $e$ then $x_{\alpha} \stackrel{u o}{\longrightarrow} x$ if and only if $\left|x_{\alpha}-x\right| \wedge e \stackrel{o}{\longrightarrow} 0$. It is clear that order convergence agrees with uo-convergence for eventually bounded nets. They agree also for sequences if the space is universally complete but they do not for nets. We mention also a very useful result obtained in [13]: If $X$ is a vector lattice, $Y$ a regular vector sublattice of $X$ and $\left(y_{\alpha}\right)$ a net in $Y$ then $y_{\alpha} \stackrel{u o}{\longrightarrow} 0$ in $Y$ if and only if $y_{\alpha} \stackrel{u o}{\longrightarrow} 0$ in $X$. This can be applied, in particular, when $X=Y^{u}$ is the universal completion of a Dedekind complete Riesz space $Y$ since in this case $Y$ is an ideal of $X$ and every ideal is regular.

The third mode of convergence can be viewed as a generalization of convergence in probability. We assume here that $X$ is equipped with a conditional expectation operator $T$ with $T e=e$. Recall that $T$ is an order continuous strictly positive projection which has $R(T)$ a Dedekind complete Riesz subspace.

Definition 3 Let $E$ be a Dedekind complete Riesz space with weak order unit $e$, and let $T$ be a conditional expectation operator on $E$ satisfying $T e=e$. We say that a net $\left(x_{\alpha}\right)_{\alpha \in A}$ converges in $T$-conditional probability to $x$, and we write $x_{\alpha} \stackrel{T P}{\longrightarrow} x$, if the net $\left(T P_{\left.\left(\left|x_{\alpha}-x\right|-\epsilon e\right)+e\right)}\right.$ converges in order to 0 for each $\epsilon>0$.

This notion has been introduced in [4] as a generalization of convergence in probability. Indeed, it agrees with the convergence in probability for sequences in the case when $X=L_{1}(\Omega, \mathcal{F}, \mathbb{P})$ and $T=\mathbb{E}$ is the expectation operator. It is perhaps worth mentioning that if $T=\operatorname{Id}_{X}$ is the identity map, then the convergence in $T$-conditionally probability is the unbounded order convergence. So, the following result is a generalization of [13, Corollary 3.5]; it could also be compared with [10, Lemma 2.11].

Lemma 4 Let $X$ be a Dedekind complete Riesz space with weak order unit $e$ and $T$ a conditional expectation with $T e=e$. For a net $\left(x_{\alpha}\right)_{\alpha \in A}$ in $X$, the following are equivalent.

(i) $x_{\alpha} \longrightarrow x$ in T-conditional probability;

(ii) $T\left(\left|x_{\alpha}-x\right| \wedge u\right) \stackrel{o}{\longrightarrow} 0$ for every $u \in X_{+}$;

(iii) $T\left(\left|x_{\alpha}-x\right| \wedge e\right) \stackrel{o}{\longrightarrow} 0$. 
Proof. Without loss of generality we may assume that $x=0$.

(i) $\Longrightarrow$ (iii) Let $\varepsilon \in(0, \infty)$.and put $P_{\varepsilon}=P_{\left(\left|x_{\alpha}\right| \wedge e-\varepsilon e\right)^{+}}$. Since $P_{\varepsilon}^{d}\left|x_{\alpha}\right| \leq \varepsilon e$ we have

$$
\begin{aligned}
\left|x_{\alpha}\right| \wedge e & =P_{\varepsilon}\left(\left|x_{\alpha}\right| \wedge e\right)+P_{\varepsilon}^{d}\left(\left|x_{\alpha}\right| \wedge e\right) \\
& \leq P_{\varepsilon} e+P_{\varepsilon}^{d}\left(\left|x_{\alpha}\right|\right) \leq P_{\varepsilon} e+\varepsilon e .
\end{aligned}
$$

Now apply $T$ to the above display and taking the limit supremum over $\alpha$ to get

$$
\limsup _{\alpha} T\left(\left|x_{\alpha}\right| \wedge e\right) \leq T \varepsilon e=\varepsilon e .
$$

As this happens for every $\varepsilon>0$ we derive that $\lim \sup T\left(\left|x_{\alpha}\right| \wedge e\right)=0$ and then $\lim T\left(\left|x_{\alpha}\right| \wedge e\right)=0$ as required.

(iii) $\Longrightarrow$ (i) Assume that $T\left(\left|x_{\alpha}\right| \wedge e\right) \stackrel{o}{\longrightarrow} 0$. Then for every $\varepsilon \in(0,1)$ we have $P_{\left(\left|x_{\alpha}\right|-\varepsilon e\right)^{+}} \leq e \leq \varepsilon^{-1} e$. On the other hand, from the inequality

$$
P_{\left(\left|x_{\alpha}\right|-\varepsilon e\right)^{+}} e \leq \varepsilon^{-1}|x|
$$

it follows that

$$
T P_{\left(\left|x_{\alpha}\right|-\varepsilon e\right)^{+}} e \leq \varepsilon^{-1} T\left(\left|x_{\alpha}\right| \wedge e\right) \stackrel{o}{\longrightarrow} 0
$$

which gives (ii).

(ii) $\Longleftrightarrow$ (iii). The forward implication is trivial. For the converse assume that (iii) occurs and let $u \in X_{+}$. Then

$$
\begin{aligned}
T\left(\left|x_{\alpha}\right| \wedge u\right) & =T\left(\left|x_{\alpha}\right| \wedge u-\left(\left|x_{\alpha}\right| \wedge u \wedge k e\right)+T\left(\left|x_{\alpha}\right| \wedge e\right)\right. \\
& \leq T(u-u \wedge k e)+T\left(\left|x_{\alpha}\right| \wedge k e\right)
\end{aligned}
$$

Hence $\lim \sup T\left(\left|x_{\alpha}\right| \wedge u\right) \leq T(u-u \wedge k e)$ for every $k>0$. Letting $k$ to $\infty$ yields $\lim \sup T\left(\left|x_{\alpha}\right| \wedge u\right)=0$, which shows that $T\left(\left|x_{\alpha}\right| \wedge u\right) \stackrel{o}{\longrightarrow} 0$ as required.

For each mode of conververgence mentioned above we may define Cauchy net to be a net $\left(x_{\alpha}\right)_{\alpha \in A}$ such that the net $\left(x_{\alpha}-x_{\beta}\right)_{(\alpha, \beta) \in A \times A}$ converges to 0 .

For more information about order convergence and unbounded order convergence the reader is referred to [13, 1] and references therein and to [4] for convergence in $T$-conditionally probability. 


\section{Properties of the sup-completion}

We consider in this section a Dedekind complete Riesz space $X$. The notion of sup-completion has been introduced by Donner in [11] and it is used there to prove some extension Theorems. It has been explored by Grobler in [14] to construct Daniell integral and then develop a kind of Functional Calculus in Riesz spaces. Some extra results have been obtained by the first author in [1] and used to get a presentation theorem of element of $X^{s}$ by integrals. This is a crucial step to prove the main theorem in [1], which states that a Riesz space is universally complete if and only if it is uo-complete. To begin the discussion, let us recall briefly the construction of the sup-completion. Consider the set $\mathcal{A}$ of all nonempty, upward directed subsets of $X$ endowed with the equivalence relation $\sim$ given by

$$
A \sim B \Longleftrightarrow \sup _{a \in A}(x \wedge a)=\sup _{b \in B}(x \wedge b) \text { for all } x \in X .
$$

Addition, multiplication by positive scalars, and ordering are defined on $\mathcal{A}$ in a natural way. Endowed with the quotient operations, the quotient space $\mathcal{A} / \sim$ is an ordered cone, called the sup-completion of $X$ and denoted by $X^{s}$. The space $X$ can be viewed as a subset of $X^{s}$ by identifying $x$ with the class of $\{x\}$ for each element $x \in X$. Theorem 1.4 in [11] provides a characterization of the cone $X^{s}$ and contains the fundamental properties of that cone. To make the reading of this paper easy we list below the most important properties of $X^{s}$, which include the extra results obtained in [1]. The reader can observe that some of these properties are stated with different assumptions, which is not easy to keep in mind (see for example, (P3) and (P6) below) and it is natural to ask whether these properties hold without any extra assumption. Our first purpose in this section is to extend these properties in more general setting by relaxing the assumptions, which makes them more natural and makes their utilization easier. The second purpose is to introduce finite and infinite parts of elements of $X^{s}$ and investigate their properties. This enables us to get satisfactory abstract formulation of some results in the setting of Riesz spaces. It is the case of the second Borel-Cantelli lemma (Theorem 29) and Theorems 35 and 36 . For any element $y \in X^{s}$, let $[y]^{\leq}$denote the subset $\{x \in X: x \leq y\}$. We are now ready to list several important properties of the cone $X^{s}$. The reader is referred to [11] and [1] for their proofs.

(P1) $X$ is the set of invertible elements in $X^{s}$ with coinciding algebraic and order structures. 
(P2) For every $y \in X^{s}$ we have $y=\sup [y]^{\leq}$.

(P3) For every $x, y \in X^{s}, a \in X$ we have

$$
x+a \wedge y=(x+a) \wedge(x+y) .
$$

(P4) If $x \in X$ and $y \in X^{s}$ satisfy $y \leq x$ then $y \in X$.

(P5) $X^{s}$ has a greatest element.

(P6) For any two non-empty subsets $A, B \subset X^{s}$ satisfying $\sup A=\sup B$ the equality

$$
\sup _{a \in A}(a \wedge x)=\sup _{b \in B}(b \wedge x)
$$

holds for every $x \in X$. In particular, if $m=\sup A$ then

$$
m \wedge x=\sup _{a \in A}(a \wedge x) .
$$

(P7) If $X$ has a weak order unit $e$ and $0 \leq x \in X^{s}$, then

$$
x=\sup _{k \geq 1}(k e \wedge x) .
$$

(P8) If $A$ and $B$ are non-empty subsets of $X$ then

$$
\sup (A+B)=\sup A+\sup B .
$$

(P9) Birkhoff Inequality: If $a, b \in X, c \in X^{s}$ then

$$
|a \wedge c-b \wedge c| \leq|a-b| .
$$

(P10) Riesz Decomposition Property: If $0 \leq x, y, z \in X^{s}$ with $x \leq y+z$ then there exist $y_{1}, z_{1} \in X^{s}$ such that $y_{1} \leq y, z_{1} \leq z$ and $\quad x=y_{1}+z_{1}$.

Properties (P1)-(P8) were proved by Donner in [11, while properties (P9), (P10) have been recently shown by the first author in [1]. The greatest element of $X$ will be denoted by $\infty$.

As we have already mentioned above, the sup-completion may be a suitable space when one wants to extend maps, especially those extensions that preserve supremum for increasing nets. This occurs when dealing with integrals of functions taking their values in $[0, \infty]$ and allow integrals to be infinite. The following result goes in this direction. It extends [1, Proposition 3] and improves it. 
Theorem 5 Let $X$ and $Y$ be two Dedekind complete Riesz spaces and $f$ : $X \rightarrow Y$ be an order continuous increasing map. Then the following statements hold.

(i) $f$ can be extended to a unique left order continuous increasing map $f^{s}$ from $X^{s}$ to $Y^{s}$. If $f$ is additive (resp. positively homogeneous), then so is $f^{s}$.

(ii) If $f$ is only defined from $X_{+}$to $Y_{+}$then $f$ has a unique left order continuous extension $f^{s}$ from $X_{+}^{s}$ to $Y_{+}^{s}$ which is increasing. Moreover, if $f$ is additive (resp. positively homogeneous), then so is $f^{s}$.

(iii) If $f$ is, in addition, a linear projection and $f(X)$ is a Riesz subspace of $Y=X$, then $f(X)$ is regular and $f^{s}\left(X^{s}\right)=f(X)^{s}$.

(iv) If $f$ is, in addition, linear and $Z$ is a regular Riesz subspace of $X$ which is invariant under $f$, then $Z^{s}$ is invariant under $f^{s}$.

Proof. The proof of (i) is exactly [1, Proposition 3] and (ii) can be proved in a similar way. Although only existence is proved in [1, Proposition 3], the ionicity is, however, obvious.

We need only to prove (iii). First we show that $f(X)$ is regular in $X$. Consider a net $\left(x_{a}\right)_{\alpha \in A}$ in $f(X)$ such that $x_{\alpha} \uparrow x$ in $f(X)$ and let $y=\sup _{\alpha \in A} x_{\alpha}$ in $X$. We have to show that $x=y$, or equivalently, $x \in f(X)$. But as $f$ is order continuous we have $x_{\alpha}=f\left(x_{\alpha}\right) \uparrow f(y)$ in $X$. This shows that $x=$ $f(y) \in f(X)$ as required. Next we will show the equality $f(X)^{s}=f^{s}\left(X^{s}\right)$. We know that if $a \in X^{s}$, then $f^{s}(a)=\sup f\left([a]^{\leq}\right) \in f(X)^{s}$ (see the proof of [1, Theorem 6]). Conversely if $u \in f(X)^{s}$ then again by [1, Theorem 6], $u=\sup f(A)$ for some upward directed subset $A$ of $X$. As $f^{s}$ is left order continuous we get $u=f^{s}(\sup A) \in f^{s}\left(X^{s}\right)$.

(iv) This is almost done in the second part of (iii).

Let $T$ be a conditional expectation operator defined on a Dedekind complete Riesz space $X$ fixing an order weak unit $e$. It follows from Theorem 5. (iii) that $R(T)$ a regular Riesz subspace of $X$ and that $R(T)^{s}=R\left(T^{s}\right)$ where $T^{s}$ denotes the extension of $T$ to $X^{s}$. It is easy seen that $T^{s} x=x$ for every $x \in R(T)^{s}$. So we have the following.

Corollary 6 Let $T$ be a conditional expectation operator defined on a Dedekind complete Riesz space $X$ fixing an order weak unit e. Then $R(T)$ is a regular Riesz subspace of $X$ and $R\left(T^{s}\right)=R(T)^{s}$. 
According to Theorem 5 , an order projection $P$ defined on $X$ extends to $X^{s}$, and its extension, denoted by $P^{s}$, satisfies $P^{s} x=\sup P x_{a}$ for every net $\left(x_{\alpha}\right)$ in $X^{s}$ such that $x_{\alpha} \uparrow x$. The following result supplies Lemma 3.1 and Theorem 3.2 in $[22$.

Proposition 7 With same notations as above, if $g \in \mathcal{R}(T)_{+}^{s}$, then $\mathcal{R}(T)^{s}$ is invariant under $P_{g}^{s}$ and $\left(P_{g}^{d}\right)^{s}$ and $B_{g}^{s}$ and $\left(B_{g}^{d}\right)^{s}$ are invariant under $T^{s}$. Moreover, $T^{s} P_{g}^{s}=P_{g}^{s} T^{s}$ and $\left(P_{g}^{d}\right)^{s} T^{s}=T^{s}\left(P_{g}^{d}\right)^{s}$.

Proof. We have $P_{g}=P_{P_{g} e}$, and $P_{g} e=\sup n g \wedge e \in R(T)$. It follows from [22, Lemme 3.1] that $P_{g} T=T P_{g}$. Observe now that $P_{g}^{s} T^{s}$ and $T^{s} P_{g}^{s}$ are tow extensions of $P_{g} T$ that are increasing and left order continuous. The fact that $B_{g}^{s}$ and $\left(B_{g}^{d}\right)^{s}$ are invariant under $T^{s}$ follows from Theorem 5 . (iv). Also the first part of this Theorem shows that $P_{g}^{s} T^{s}=T^{s} P_{g}^{s}$ as both of them are increasing and left order continuous and extend $T P_{g}$. Similarly we show that $\left(P_{g}^{d}\right)^{s} T^{s}=T^{s}\left(P_{g}^{d}\right)^{s}$.

Our next goal is to extend some properties of $X^{s}$ obtained earlier by Donner or by the first author. We will extend property (P10) by showing that the Riesz decomposition property is valid for all elements of $X^{s}$. Recall that for every $x \in X^{s}$ one can define its positive and negative parts repectively by $x^{+}=x \vee 0$ and $x^{-}=-(x \wedge 0)$ and that $x^{-} \in X$.

Lemma 8 Let $x, y, z \in X^{s}$ such that $x \leq y+z$. then there exist $y_{1}, z_{1} \in X^{s}$ such that

$$
y_{1} \leq y, z_{1} \leq z \quad \text { and, } \quad x=y_{1}+z_{1} .
$$

If, in addition, $x \in X$ then $y_{1}, z_{1} \in X$.

Proof. Assume that $x \leq y+z$. Then $u=x^{+}+y^{-}+z^{-} \leq y^{+}+z^{+}+x^{-}$. By the Riesz decomposition property in $X_{+}^{s},(\mathrm{P} 10)$, one can write $u=a+b+c$ with

$$
0 \leq a \leq y^{+}, 0 \leq b \leq z^{+}, 0 \leq c \leq x^{-} .
$$

So $x=y_{1}+z_{1}$ is a required decomposition as $y_{1}=a-y^{-} \leq y$ and $z_{1}=$ $b-z^{-}+c-x^{-} \leq z$. Now, if $x \in X$ then $u \in X$ and so $a, b, c \in X$ by (P4). It follows that $y_{1}, z_{1} \in X$, which completes the proof.

Corollary 9 Let $A$ and $B$ be two subsets of $X^{s}$. Then $\sup (A+B)=\sup A+$ $\sup B$. 
Proof. The inequality $\sup (A+B) \leq \sup A+\sup B$ is obvious. To show the converse inequality it is enough to prove the following inclusion

$$
[\sup A+\sup B]^{\leq} \subseteq[\sup (A+B)]^{\leq} .
$$

To this end, let $u \in[\sup A+\sup B]^{\leq}$and use the Riesz decomposition property to write $u=x+y$ with $x \leq \sup A$ and $y \in \sup B$. Pick $a \in A, b \in B$ and observe that

$$
x \wedge a+y \wedge b \leq a+b \leq \sup (A+B) .
$$

Using properties (P8) and (P6) to get

$$
\sup _{a \in A, b \in B}(x \wedge a+y \wedge b)=\sup _{a \in A}(x \wedge a)+\sup _{b \in B}(y \wedge b)=x+y=u .
$$

We deduce that $u \in[\sup (A+B)]^{\leq}$, and this completes the proof.

Remark 10 If $E$ and $F$ are two Dedekind complete Riesz spaces then $E \times F$ is a Dedekind complete Riesz space with $(E \times F)_{+}=E_{+} \times F_{+}$. Moreover it is not hard to see that $(E \times F)^{s}=E^{s} \times F^{s}$ and then $(E \times F)_{+}^{s}=E_{+}^{s} \times F_{+}^{s}$. This fact combined with Proposition 14 will help us to get quick proofs of several properties of $X^{s}$.

Lemma 11 Let $\left(x_{\alpha}\right)$ and $\left(y_{\beta}\right)$ be two nets in $X_{+}^{s}$ and let $x, y \in X_{+}^{s}$. such that $x=\sup x_{\alpha}$ and $y=\sup y_{\beta}$. Then the following hold.

(i) $x+y=\sup \left(x_{\alpha}+y_{\beta}\right)$.

(ii) $x \vee y=\sup \left(x_{\alpha} \vee y_{\beta}\right)$.

(iii) $x \wedge y=\sup \left(x_{\alpha} \wedge y_{\beta}\right)$.

(iv) If $x \wedge y=0$ then $x+y=x \vee y$.

(v) $x+y=x \vee y+x \wedge y$.

(vi) For every $a \in X_{+}^{s}$ we have

$$
a+x \wedge y=(a+x) \wedge(a+y), \text { and } a+x \vee y=(a+x) \vee(a+y)
$$


Proof. (i) follows from (P8) if the nets $\left(x_{\alpha}\right)$ and $\left(y_{\beta}\right)$ are chosen in $X$. For the general case we use Corollary 9 .

(ii) It is clear that $x \vee y \geq \sup _{\alpha, \beta} x_{\alpha} \vee y_{\beta}$. On the other hand the inequalities $\sup _{\alpha, \beta}\left(x_{\alpha} \vee y_{\beta}\right) \geq \sup _{\alpha} x_{\alpha}=x$ and $\sup _{\alpha, \beta}\left(x_{\alpha} \vee y_{\beta}\right) \geq y$ are also obvious and then $\sup _{\alpha, \beta}\left(x_{\alpha} \vee y_{\beta}\right) \geq x \vee y$, which gives (ii).

(iii) The inequality $\sup \left(x_{\alpha} \wedge y_{\beta}\right) \leq x \wedge y$ is clear. For the converse we will assume first that $\left(x_{\alpha}\right)$ and $\left(y_{\beta}\right)$ are in $X$. Let $u \geq x_{\alpha} \wedge y_{\beta}$ and let $z \in[x \wedge y]^{\leq}$, which means that $z \in X, z \leq x$ and $z \leq y$. Then $u \geq z \wedge x_{\alpha} \wedge y_{\beta}$. As this happens for every $\beta$ we get by (P6), $u \geq z \wedge x_{\alpha} \wedge y=z \wedge x_{\alpha}$. Using once more (P6) we obtain $u \geq z \wedge x=z$. As this happens for every $z \in[x \wedge y]^{\leq}$ it follows from (P2) that $u \geq x \wedge y$ and then $\sup \left(x_{\alpha} \wedge y_{\beta}\right)=x \wedge y$.

The general case can be derived from the above case and Theorem 5 applied to the map

$$
f: X_{+} \times X_{+} \longrightarrow X_{+} ;(x, y) \longmapsto x \wedge y .
$$

(see Remark [10).

(iv) Clearly, $x \vee y \leq x+y$. Let $z \in[x+y]^{\leq}$then there exist $x^{\prime} \in[x]^{\leq}$ and $y^{\prime} \in[y]^{\leq}$such that $z=x^{\prime}+y^{\prime}$. But as $x^{\prime} \perp y^{\prime}$ we get $z=x^{\prime}+y^{\prime}=$ $x^{\prime} \vee y^{\prime} \leq x \vee y$, which gives the converse inequality: $x+y \leq x \vee y$.

(v) and (vi) follow from (i), (ii) and (iii) above and (P2).

Lemma 12 Let $x, y, z \in X_{+}^{s}$. Then

(i) $x \wedge(y+z) \leq x \wedge y+x \wedge z$.

(ii) If, in addition, $y \wedge z=0$ then $x \wedge(y+z)=x \wedge y+x \wedge z$.

(iii) If $x \wedge z=0$ then $x \wedge(y+z)=x \wedge y$.

Proof. (i) It follows easily from the Riesz decomposition property in $X^{s}$ that for every $0 \leq u \in[x \wedge(y+z)]^{\leq}$there exist $a, b \in X$ such that $0 \leq a \leq y$, $0 \leq b \leq z$ and $u=a+b$. Moreover as $a, b \leq x$ we get $a \leq x \wedge y$ and $b \leq x \wedge z$, and then $u \leq x \wedge y+x \wedge z$. We deduce now from (P2) that

$$
x \wedge(y+z) \leq x \wedge y+x \wedge z .
$$

(ii) In the case when $y \wedge z=0$ we use Lemma 11.(iv) to get

$$
x \wedge(y+z)=x \wedge(y \vee z)=(x \wedge y) \vee(x \wedge z)=(x \wedge y)+(x \wedge z) .
$$


(iii) This follows easily from the Riesz decomposition property in $X^{s}$ and property (i) above.

Corollary 13 Let $x, y, v, w \in X_{+}^{s}$ such that $x \perp y$ and $v \perp w$. Then

$$
(x+v) \wedge(y+w)=x \wedge w \stackrel{\perp}{+} y \wedge v .
$$

Proof. It is clear that $x \wedge w+y \wedge v$ is a lower bound of $\{x+v, y+w\}$. On the other hand by Lemma 12 we have

$$
(x+v) \wedge(y+w) \leq x \wedge(y+w)+v \wedge(y+w)=x \wedge w+v \wedge y
$$

which yields the desired equality.

It is worth noting that if we define a map $\varphi: X \times X \longrightarrow X$ by putting $\varphi(x)=x \vee y$ then with notation of Theorem 5, the second assertion in Lemma 11 means that $\varphi^{s}(x, y)=x \vee y$. In a similar way properties (i) and (iii) in the same lemma can be interpreted. The following result gives us another way to prove the other properties of Lemma 11. It will be also used in the sequel to get quick proofs.

Proposition 14 All spaces in the following statements are assumed to be Dedekind complete and maps order continuous and increasing.

(i) If $f, g: X \longrightarrow Y$ satisfy $f \leq g$ then $f^{s} \leq g^{s}$.

(ii) If $f, g, h: X \longrightarrow Y$ satisfy $h=f+g$ then $h^{s}=f^{s}+g^{s}$.

(iii) If $f: X \longrightarrow Y$ and $g: Y \longrightarrow Z$ then $(g \circ f)^{s}=g^{s} \circ f^{s}$.

Proof. The proof of (i) is trivial. (ii) is very similar to Lemma 11, Let us prove (iii). Let $x \in X^{s}$ and let $\left(x_{\alpha}\right)$ a net in $X$ such that $x_{\alpha} \uparrow x$. Then $f\left(x_{\alpha}\right)=f^{s}\left(x_{\alpha}\right) \uparrow f^{s}(x)$. So $g^{s}\left(f^{s}\left(x_{\alpha}\right)\right)=g\left(f\left(x_{\alpha}\right)\right) \uparrow g^{s}\left(f^{s}(x)\right)$. On the other hand $g^{s}\left(f^{s}\left(x_{\alpha}\right)\right)=(g \circ f)\left(x_{\alpha}\right) \uparrow(g \circ f)^{s}(x)$. This yields the desired equality $(g \circ f)^{s}(x)=g^{s}\left(f^{s}(x)\right)$. 


\subsection{Finite and infinite parts of an element in $X^{s}$.}

It was shown in [1] that if $Y$ is a regular Riesz subspace of a Dedekind complete Riesz space $X$ then the subset of $X^{s}$ defined by

$$
Z=\{\sup A: \emptyset \neq A \subseteq Y\}
$$

is the sup-completion of $Y$. In particular, if $Y$ is an ideal of $X$ then $Y^{s} \subseteq X^{s}$. We will denote by $\infty_{Y}$ the greatest element of $Y$. If $X=\mathbb{R}^{\Omega}$ is the Riesz space of all real valued functions defined on a set $\Omega$, then $X^{s}=\mathbb{R}_{\infty}^{\Omega}$ is consisting of all functions defined on $\Omega$ taking values in $\mathbb{R}_{\infty}=\mathbb{R} \cup\{\infty\}$. Every element in $X^{s}$ can be decomposed as follows: $f=f \cdot \chi_{A}+\infty \cdot \chi_{\mathbb{R} \backslash A}$, where $A=\{x \in \Omega: f(x) \in \mathbb{R}\}$. We can say that $f \cdot \chi_{A}$ is the finite part of $f$ and $\infty \cdot \chi_{\mathbb{R} \backslash A}=f \chi_{\mathbb{R} \backslash A}$ is its infinite part. A similar decomposition can be obtained in the case when $X=L_{p}(\mu)$ with $1 \leq p \leq \infty$. The goal of this subsection is to extend this decomposition to a general Dedekind complete Riesz space.

Theorem 15 Let $x \in X_{+}^{s}$. Then there is a unique projection band $B$ in $X$ such that $x=\infty_{B}+u$ with $u \perp B$ in $X^{u}$.

Proof. Let $B$ denote the band of $X$ generated by the subset

$$
\{u \in X: x \geq t|u| \text { for all } t \in(0, \infty)\}
$$

and let $P$ be the corresponding band projection. We extend $P$ and $P^{d}=I-P$ to $X^{s}$. It is easily seen that $P^{s} x=\sup H=\sup B$ is the largest element of $B^{s}$ and that $\left(P^{d}\right)^{s} x \in X$. Also, it is not difficult to see that the set defined in (11) is in fact a band in $X$ and then it coincides with $B$. Let us denote this set by $H$. We will only check that $H$ is closed under addition. To this end, take $a, b$ in $H$. Then, by definition, $x \geq 2 t|a|$ and $x \geq 2 t|b|$ for every $t \geq 0$. It follows that $x \geq \frac{1}{2}(2 t|a|+2 t|b|) \geq t|a+b|$ and hence $a+b \in H$ as claimed. We now observe that for each $a \in B_{+}$, we have

$$
x \geq P^{s} x \geq P^{s} a=P a=a .
$$

This shows that $P^{s} x=\infty_{B}$. We continue to note the band projection on the band generated by $B$ in $X^{u}$ by $P$ and we claim that $P^{d} x \in X^{u}$. Otherwise it follows from [1, Corollary 15] that there exists $a \in B_{+}^{d}$ such that $P^{d} x \geq t a>0$ for all real $t \geq 0$. This clearly implies that $a \in H=B$, a contradiction. 
It remains to show that the above decomposition is the unique one. Assume that $x=\infty_{C}+v$ is another decomposition with $v \perp C^{d}$. Then for each $a \in C$, we have $x=\infty_{C}+v \geq t|a|$ for every $t \in \mathbb{R}_{+}$. This shows that $C$ is contained in $B$. Conversely if $a \in B$ then $\left(P_{C}^{d}\right)^{s} x=v \geq t P_{C}^{d} a$ for ever $t \in(0, \infty)$ and so $P_{C}^{d} a=0$, as $X$ is Archimedean. This shows that $a \in C$ and completes the proof.

Definition 16 Let $x \in X_{+}^{s}$. Then $\infty_{B}$ and $u$ defined in Theorem 15 are the infinite part and finite part of $x$. They will be denoted by $x^{\infty}$ and $x^{f}$, respectively.

The following is an immediate consequence of the definition above and it will be useful later on.

Corollary 17 Let $X$ be a Dedekind complete Riesz space and $x \in X_{+}^{s}$. Then $P \perp P_{x^{\infty}}$ if and only if $P x \in X^{u}$.

In [1] the first author has shown the following result which provides a characterization of elements of the sup-completion of a Dedekind complete Riesz space that do not belong to its universal completion.

Theorem 18 ([1, Theorem 14]) Let $X$ be a Dedekind complete Riesz space with weak order unit $e$ and let $x \in X$. Then

$$
x \in X^{s} \backslash X^{u} \Longleftrightarrow \bigwedge_{k=1}^{\infty} P_{(x-k e)^{+}} e>0 .
$$

Now, having defined the finite and infinite parts in $X^{s}$ we can prove a more precise result.

Theorem 19 Let $X$ be a Dedekind complete Riesz space with weak order unit $e$ and let $x \in X_{+}^{s}$. Then $\bigwedge_{k=1}^{\infty} P_{(x-k e)^{+}} e=P_{x^{\infty}} e$.

Proof. Write $x=x^{\infty}+x^{f}$ and let $P=P_{x^{\infty}}$ be the band projection on $X^{u}$. Observe that

$$
x-k e=x^{\infty}-k P e+x^{f}-k P^{d} e=x^{\infty}+x^{f}-k P^{d} e .
$$


Thus $(x-k e)^{+}=x^{\infty}+\left(x^{f}-k P^{d} e\right)^{+}$. Since $x^{\infty} \wedge\left(x^{f}-k P^{d} e\right)^{+}=0$, it follows that

$$
P_{(x-k e)^{+}}=P_{x^{\infty}}+P_{\left(x^{f}-k P^{d} e\right)^{+}}=P_{x^{\infty}} \vee P_{\left(x^{f}-k P^{d} e\right)^{+}},
$$

which implies that

$$
\bigwedge_{k=1}^{\infty} P_{(x-k e)^{+}}=P_{x^{\infty}} \vee \bigwedge_{k=1}^{\infty} P_{\left(x^{f}-k P^{d} e\right)^{+}}=P_{x^{\infty}}
$$

where the last equality follows from Theorem 18 above as $P^{d} e$ is a weak order unit in the band $B_{x^{\infty}}^{d}$.

A more general statement, which can be deduced from Theorem 19, is the following:

Corollary 20 Let $X$ be a Dedekind complete Riesz space with order weak unit. If $x \in X_{+}^{s}$ and $u \in X_{+}$. then

$$
\bigwedge_{k=1}^{\infty} P_{(x-k u)^{+}}=P_{P_{u}^{s} x^{\infty}}+P_{\left(P_{u}^{d}\right)^{s} x}=P_{u} P_{x^{\infty}}+P_{u}^{d} P_{x} .
$$

The following result is easy to prove and will be used in the proof of Theorem 36 .

Proposition 21 Let $X$ be a Dedekind complete Riesz space $x, y \in X_{+}^{s}$ and $\lambda \in \mathbb{R}_{+}$Then the following hold:

1. $(x+y)^{\infty}=x^{\infty}+y^{\infty}$ and $(\lambda x)^{\infty}=\lambda x^{\infty}$

2. If $x \leq y$ then $x^{\infty} \leq y^{\infty}$;

3. $(x \vee y)^{\infty}=x^{\infty} \vee y^{\infty}$ and $(x \wedge y)^{\infty}=x^{\infty} \wedge y^{\infty}$.

We end this section with a result which will be needed later in the proof of Theorem 29, 
Lemma 22 Let $X$ be a Dedekind complete Riesz space and let $\left(x_{n}\right)$ be a sequence in $X_{+}^{u}$. Assume that $\sum_{n=1}^{\infty} x_{n}=\infty_{B}+u$ as in Proposition 15 and let

$$
\begin{aligned}
R_{n}=\sum_{k=n}^{\infty} x_{k} . \text { Then for every } y & \in X_{+}, \\
P y & =\lim \left(y \wedge R_{n}\right),
\end{aligned}
$$

where $P$ denotes the band projection on $B$.

Proof. Observe first that $P^{d} \sum_{n=1}^{\infty} x_{n}=\sum_{n=1}^{\infty} P^{d} x_{n}=u$. It follows that $P^{d} R_{n}=$ $\sum_{k=n}^{\infty} P^{d} x_{n} \stackrel{o}{\longrightarrow} 0$. On the other hand we deduce from Lemma 12 that

$$
y \wedge R_{n}=y \wedge\left(P R_{n}+P^{d} R_{n}\right)=y \wedge P R_{n}+y \wedge P^{d} R_{n} .
$$

As $P R_{n}=\infty_{B}$ for every $n$ and $y \wedge P^{d} R_{n} \stackrel{o}{\longrightarrow} 0$, the result follows.

\subsection{Multiplication in $\mathrm{X}^{s}$}

Consider again a Dedekind complete Riesz space $X$ with weak order unit $e$. We know that the universal completion $X^{u}$ of $X$ is equipped with an $f$-algebra multiplication with $e$ as identity. Our aim in this subsection is to extend the multiplication to the positive part of the cone $X^{s}$ and prove several properties of that multiplication which extend standard ones. Recall, by the way, that although $X^{u}$ is not contained in $X^{s}$ in general, its positive cone $X_{+}^{u}$ does ([1, Corollary 7]). We defined the product of two elements $x$ and $y$ of $X_{+}^{s}$ as follows:

$$
\begin{aligned}
x y & =\sup \left\{v w: 0 \leq v \in[x]^{\leq}, 0 \leq w \in[y]^{\leq}\right\} \\
& =\sup \left\{v w: 0 \leq(v, w) \in[(x, y)]^{\leq}\right\} .
\end{aligned}
$$

Thus the product on $X_{+}^{s}$ is the unique extension of the product on $X_{+}$in the sense of Theorem 5, (ii). By considering the map

$$
\pi: X_{+} \times X_{+} \longrightarrow X_{+}^{u} ;(x, y) \longmapsto x y
$$

one can see that if $x, y \in X_{+}^{+}$then $x y=\pi^{s}(x, y)$. The following result is then an immediate consequence of Theorem 5 , 
Lemma 23 Let $\left(x_{\alpha}\right),\left(y_{\beta}\right)$ be two nets in $X_{+}^{s}$ such that $x_{\alpha} \uparrow x$ and $y_{\beta} \uparrow y$. Then $x_{\alpha} y_{\beta} \uparrow x y$.

As expected the product on $X^{s}$ shares some standard properties.

Lemma 24 Let $x, y, z \in X_{+}^{s}$. The following statements hold.

(i) $x(y+z)=x y+x z$.

(ii) $x(y \wedge z)=x y \wedge x z$.

(iii) $x(y \vee z)=x y \vee x z$.

Proof. (i) Define the following maps

$$
\begin{aligned}
& h: X_{+} \times X_{+} \times X_{+} \longrightarrow X_{+} ;(x, y, z) \longmapsto x(y+z) ; \\
& f: X_{+} \times X_{+} \longrightarrow X_{+} ;(x, y) \longmapsto x y ; \\
& g: X_{+} \times X_{+} \longrightarrow X_{+} ;(x, z) \longmapsto x z .
\end{aligned}
$$

All of them are increasing and order continuous and satisfy $h=f+g$. Apply then Proposition 14 to obtain the equality $h^{s}=f^{s}+g^{s}$, which means exactly the required equality.

(ii) and (iii) can be proved in a similar way.

We list next some properties of the product on $X_{+}^{s}$.

Proposition 25 Let $X$ be a Dedekind complete Riesz space. $B$ and $C$ be two bands in $X$ and $x, y \in X_{+}^{s}$. The following properties hold.

(i) If $x \in X_{+}^{s}$ and $B \in \mathfrak{B}(X)$ then $x . \infty_{B}=\infty_{P_{B x}}$. In particular, $x . \infty=$ $\infty_{B_{x}}$ and $\left(x . \infty_{B}\right)^{f}=0$.

(ii) If $B, C \in \mathcal{B}(X)$ then, $\infty_{B} \wedge \infty_{C}=\infty_{B} \cdot \infty_{C}=\infty_{B \cap C}$ and $\infty_{B} \vee \infty_{C}=$ $\infty_{B}+\infty_{C}=\infty_{B+C}$.

(iii) If $x, y \in X_{+}^{s}$ then $(x y)^{f}=x^{f} y^{f}$ and $(x y)^{\infty}=x^{\infty} y^{\infty}+x^{\infty} y^{f}+x^{f} y^{\infty}$.

Proof. (i) It follows from the definition of the product in $X_{+}^{s}$ that $e x=x$ for all $x \in X_{+}^{s}$. On other hand Property (P7) yields that if $0<u \in X$ is a weak order unit then $\sup _{k} k u=\infty$. Thus

$$
\infty x=\sup k e \cdot x=\sup _{k \geq 1} k x=\infty_{B_{x}} .
$$


for all $x \in X_{+}^{s}$. So, if $x_{\alpha} \uparrow \infty$ and $x \in X_{+}^{s}$ then $x_{\alpha} x \uparrow \infty_{B_{x}}$.

(ii) Observe first that for each $n \in \mathbb{N}$ we have

$$
n P_{B} e \wedge n P_{C} e=n\left(P_{B} e \wedge P_{C} e\right)=n P_{B} e P_{C} e=n P_{B \cap C} e .
$$

Moreover, as $P_{D} e$ is a weak unit in $D$ for every band $D$, the result follows by taking the supremum over $n$ and using Lemma 11(iii). For the second formula observe that

$$
n P_{B} e \vee n P_{C} e=n P_{B+C} e \leq n P_{B}+n P_{C} e .
$$

By taking the supremum over $n$ we get

$$
\infty_{B} \vee \infty_{C}=\infty_{B+C} \leq \infty_{B}+\infty_{C}
$$

On the other hand since $\infty_{B}, \infty_{C} \in(B+C)^{s}$ we obtain $\infty_{B}+\infty_{C} \leq \infty_{B+C}$, which gives the equality.

(iii) can be deduce easily from (i).

\section{Borel-Cantelli Lemmas}

We will assume throughout this section that $X$ is a Dedekind complete Riesz space with conditional expectation operator $T$ and weak order unit $e=T e$. We recall that the space $X$ is called $T$-universally complete if every increasing net $\left(x_{\alpha}\right)$ in $X$ with $\left(T x_{\alpha}\right)$ order bounded in $X^{u}$ is order convergent in $X$. By extending $T$ to its natural domain $L^{1}(T)$ we may assume that $X$ is $T$ universally complete (see [22]). The first Borel-Cantelli Lemma has been generalized to the setting of Riesz spaces in [25]. We provide here a slight more general form of it.

Lemma 26 Let $\left(x_{n}\right)$ be an order bounded sequence of $X$. If $\sum_{n=1}^{\infty} T x_{n} \in X^{u}$, then $\lim \sup x_{n}=0$.

Remark 27 If we assume only that $T$ is positive (and not strictly positive), then we can only conclude that $T\left(\lim \sup x_{n}\right)=0$.

If $x_{n}=P_{n}$ e for some band projection $P_{n}$ we get a version of BorelCantelli Lemma.

If $\sum_{n=1}^{\infty} T P e_{n} \in X^{u}$, then $\limsup _{n \longrightarrow \infty} P e_{n}=0$. 
Proof. Since $X$ is $T$-universally complete it follows that the series $\sum x_{n}$ is order convergent in $X$. Now, as $T$ is order continuous we have

$$
T \sup _{k \geq n} x_{k} \leq T\left(\sum_{k=n}^{\infty} x_{k}\right)=\sum_{k=n}^{\infty} T x_{k},
$$

and then

$$
T\left(\limsup _{n \longrightarrow \infty} x_{n}\right)=\lim T \sup _{k \geq n} x_{k}=0 .
$$

As $T$ is strictly positive we deduce that $\limsup _{n \rightarrow \infty} x_{n}=0$, as required.

The following lemma is needed in the proof of the second Borel-Cantelli Lemma in Riesz spaces. Before stating the lemma we recall that the ideal $X_{e}$ generated by $e$ can be endowed with a multiplication for which $e$ is a unit element. If $x \in X_{e}$ and $f$ is continuous then $f(x)$ is well defined ([2, Lemma $2])$. Moreover it is easily seen that $(f g)(x)=f(x) g(x)$. One can also use the $C(K)$-representation of $X_{e}$, when the constant function 1 corresponds to the unit $e$. It should be noted that if two real functions $f$ and $g$ satisfies $f \leq g$ and $f(x)$ and $g(x)$ exist then $f(x) \leq g(x)$. As an example of this we get the inequality $e-x \leq \exp (-x)$ for $x \in X_{+}$, which will be needed later. Functional calculus done on the ideal $X_{e}$ can be extended using Daniell Integral to the whole of $X$. This allows to consider more general functions which do not need to be continuous (see [14]). For more information about functional calculus the reader is referred to [14] and [6], where he can find a comparison between the two kinds mentioned above. In the sequel we need only the following fact: If $x \in X$ then $\exp (x)$ and $\exp (-x)$ are well defined and $\exp (x) \exp (-x)=e$.

1. Lemma 28 Let $\left(x_{\alpha}\right)$ be a net in $X_{+}$such that $x_{\alpha} \uparrow x \in X_{+}^{s}$. Then $\exp \left(-x_{\alpha}\right) \downarrow$ $\exp \left(-x^{f}\right)$ where $x^{f}$ is the finite part of $x$ defined above. In particular if $x_{\alpha} \uparrow \infty$, then $\exp \left(-x_{\alpha}\right) \downarrow 0$.

Proof. Assume first that $x_{\alpha} \uparrow \infty$. Then $\left(\exp \left(-x_{\alpha}\right)\right)$ is a positive decreasing net and we have to show that its infimum is zero. If not, there exists $u>0$ such that $\exp \left(-x_{\alpha}\right) \geq u$ for every $\alpha$. In this case we get

$$
e=\exp \left(x_{\alpha}\right) \exp \left(-x_{\alpha}\right) \geq \exp \left(x_{\alpha}\right) \cdot u \geq x_{\alpha} u .
$$

Using Lemma 23 we deduce that $e \geq u^{\infty}$. But $u^{\infty} \notin X^{u}$ and this contradicts Property (P4). 
Theorem 29 (Borel-Cantelli Lemma) Let $\left(P_{n}\right)$ be a sequence of T-independent band projections in $X$. If $B$ is a band of $X^{u}$ such that

$$
\sum_{n \geq 1} T P_{n} e=\infty_{B}+u \text { with } u \in B^{d},
$$

then $P_{B}$ commutes with $T$ and

$$
P_{B}=\limsup _{n \longrightarrow \infty} P_{n} .
$$

In particular, if $\sum_{n \geq 1} T P_{n} e=\infty$ then $\limsup _{n \longrightarrow \infty} P_{n}=I$.

Proof. We will show that $P e \in \mathcal{R}(T)$ and conclude by [22, Lemma 3.1] that $T P=P T$. To this end observe first that

$$
e \wedge \sum_{k=n}^{\infty} T P_{k} e=\lim _{N \longrightarrow \infty} e \wedge \sum_{k=n}^{N} T P_{k} e \in \mathcal{R}(T) .
$$

According to Lemma 22 we deduce that $P e \in \mathcal{R}(T)$ as required.

By the first Borel-Cantelli Lemma (26) we have $P^{d} \lim \sup P_{n} e=0$. So by considering the band $B$ instead of $X$ and $P P_{n}$ instead of $P_{n}$ we may assume that $B=X$ and $\sum_{n=1}^{\infty} T P_{n} e=\infty$. We have to show that $\lim \sup P_{n}=I$, or equivalently $\left(\lim \sup P_{n}\right)^{d} e=0$. Now observe that

$$
\begin{aligned}
\left(\limsup P_{n}\right)^{d} & =\liminf P_{n}^{d}=\sup _{n}\left(\inf _{k \geq n} P_{k}^{d}\right) \\
& =\sup _{n}\left(\inf _{m \geq n} P_{n}^{d} \ldots P_{m}^{d}\right) .
\end{aligned}
$$

Since the projections $P_{n}$ are $T$-independent we have

$$
T P_{n}^{d} \ldots P_{m}^{d} e=\prod_{k=n}^{m}\left(e-T P_{k} e\right) \leq \exp \left(-\sum_{k=n}^{m} T P_{k} e\right) .
$$

Using Lemma 28 we can see that $T P_{n}^{d} \ldots P_{m}^{d} e \downarrow 0$ as $m \longrightarrow \infty$. Since all those considering sequences are increasing or decreasing the order continuity of $T$ allows us to deduce that

$$
T\left(\lim \sup P_{n}\right)^{d} e=0 .
$$

As $T$ is strictly positive we obtain $\left(\lim \sup P_{n}\right)^{d} e=0$ as required. 


\section{Applications to Borel Cantelli lemmas}

We will assume also in this section that our space $X$ is Dedekind complete Riesz space with conditional expectation operator $T$ and weak order unit $e=T e$.

The following observation may be useful. It could be compared with [19, Lemma 1.6] and [20, Lemma 1.2].

Lemma 30 Assume that $\left(x_{\alpha}\right)$ is a positive decreasing net in $X$ such that $x_{\alpha} \stackrel{T P}{\longrightarrow} 0$. Then $x_{\alpha} \downarrow 0$.

Proof. Let $x=\inf x_{\alpha}$. By Lemma 4, we have $T\left(x_{\alpha} \wedge e\right) \longrightarrow 0$. On the other hand by order continuity of $T, T\left(x_{\alpha} \wedge e\right) \downarrow T(x \wedge e)$. It follows from the strict positivity of $T$ that $x \wedge e=0$ and then $x=0$ as required.

Next we present some applications to Borel-Cantelli Lemmas in Riesz spaces. We need the following lemma.

Lemma 31 Let $\left(x_{n}\right)_{n \geq 1}$ be a sequence in $X$ such that $\left(\sup _{k \geq 0}\left|x_{n+k}-x_{n}\right|\right)_{n \geq 1}$ converges in $T$-conditional probability to 0 as $n \longrightarrow \infty$, then $\left(x_{n}\right)_{n \geq 1}$ is order convergent in $X^{u}$.

Proof. We have to show that $\delta_{n}=\sup _{p, q \geq n}\left|x_{p}-x_{q}\right| \stackrel{o}{\longrightarrow} 0$. As order convergence and uo-convergence agree for sequences in $X^{u}$ (see [21, Theorem 3.2]), it is sufficient to prove that $\sup _{p, q>n}\left|x_{p}-x_{q}\right| \wedge e \stackrel{o}{\longrightarrow} 0$ ([1, Theoem 28]) Now it follows from the assumption and Lemma 4 that $T\left(\sup _{p, q \geq n}\left|x_{p}-x_{q}\right| \wedge e\right) \stackrel{o}{\longrightarrow} 0$ and it follows from Lemma 30 that $\sup _{p, q \geq n}\left|x_{p}-x_{q}\right| \wedge e \stackrel{o}{\longrightarrow} 0$, which completes the proof.

Borel-Cantelli lemma can be used to prove almost surely convergence of sequences of random variables by showing the convergence of some series. Similar results can be obtained in the setting of Riesz spaces.

Proposition 32 Let $\left(x_{n}\right)$ be a sequence in $X$ and let $x \in X$.

(i) If the series $\sum_{n=1}^{\infty} T P_{\left(\left|x_{n}-x\right|-\varepsilon e\right)^{+}}$e is order convergent in $X^{u}$ for all $\varepsilon>0$ then $x_{n} \stackrel{\text { uo }}{\longrightarrow} x$. 
(ii) If $\sum_{n=1}^{\infty} T\left|x_{n}-x\right|^{r} \in X^{u}$ for some real $r>0$ then $x_{n} \stackrel{\text { uo }}{\longrightarrow} x$.

Proof. (i) By the first Borel-Cantelli lemma (Lemma 26), we have

$$
\limsup _{n \longrightarrow \infty} P_{\left(\left|x_{n}-x\right|-\varepsilon e\right)^{+}}=0 \quad \varepsilon>0 .
$$

So $P_{\left(\left|x_{n}-x\right|-\varepsilon e\right)+} \stackrel{o}{\longrightarrow} 0$. The result now follows from [21, Theorem 2.8].

(ii) According to Chebychev Lemma in Riesz space (see [6, Theorem 3.9]), we have

$$
T P_{\left(\left|x_{n}-x\right|-\varepsilon e\right)^{+}} e \leq \varepsilon^{-r} T\left|x_{n}-x\right|^{r} .
$$

The result follows now from (i).

It was proved in [5], that if $\left(P_{n}\right)$ is a sequence of band projections satisfying $P_{n} \uparrow I$ and if $\left(x_{n}\right)$ is a sequence in $X$ such that for every $k,\left(P_{k} x_{n}\right)_{n>1}$ is order convergent in $X^{u}$, then the sequence $\left(x_{n}\right)$ is order convergent in $X^{u}$. We need here a more general statement.

It was shown in [5] that if $X$ is a Dedekind complete vector lattice, $\left(P_{\gamma}\right)_{\gamma \in \Gamma}$ is a net of band projections such that $P_{\gamma} \uparrow I$ and $\left(x_{\alpha}\right)_{\alpha \in A}$ is a net of elements in $X$ such that for each $\gamma$ the net $\left(P_{\gamma} x_{\alpha}\right)_{\alpha \in A}$ is order convergent, then $\left(x_{\alpha}\right)$ is $u o$-convergent in $X^{u}$. Next we state a more general result: first, the order convergence of the net $\left(P_{\gamma} x_{\alpha}\right)_{\alpha \in A}$ is relaxed to $u o$-convergence, second, the net $\left(P_{\gamma}\right)$ is not assumed to be increasing.

Theorem 33 Let $\left(P_{\gamma}\right)_{\gamma \in \Gamma}$ be a net of band projections and $\left(x_{\alpha}\right)_{\alpha \in A}$ a net of elements in $X$. If for each $\gamma$, the net $\left(P_{\gamma} x_{\alpha}\right)$ is uo-convergent in $X$, then $\left(P x_{\alpha}\right)$ is uo-convergent in $X^{u}$, where $P=\sup P_{\gamma}$.

Proof. By considering the band generated by $R\left(P_{\gamma}\right), \gamma \in \Gamma$, we may assume that $P=I$. Let $\mathcal{F}$ be the set of nonempty finite subsets of $\Gamma$. For every $F \in \mathcal{F}$ we consider the band projection $P_{F}=\sup _{\gamma \in F} P_{\gamma}$. Then $\left(P_{F}\right)_{F \in \mathcal{F}}$ is an increasing net of band projections and $P_{F} \uparrow I$.

Step 1. We will show first that $\left(P_{F} x_{\alpha}\right)_{\alpha \in A}$ is uo-convergent. This can be done by induction on $k=|F|$. It is exactly what says the assumption of $k=1$. Now observe that if $F=G \cup\{\gamma\}$ with $\gamma \notin G$ then

$$
P_{F}=P_{G}+P_{G}^{d} P_{\gamma}
$$


As $\left(P_{\gamma} x_{\alpha}\right)_{\alpha \in A}$ is uo-convergent, it follows easily that $\left(P_{G}^{d} P_{\gamma} x_{\alpha}\right)_{\alpha \in A}$ is uoconvergent. So, if we assume that $\left(P_{G} x_{\alpha}\right)_{\alpha \in A}$ is uo-convergent, then the above equality can be used to conclude that $\left(P_{F} x_{\alpha}\right)_{\alpha \in A}$ is uo-convergent as well. This proves our claim.

Step 2. We will show now that $\left(x_{\alpha}\right)_{\alpha \in A}$ is uo-convergent in $X^{u}$. According to [1, Theorem 17] it is sufficient to show that $\left(x_{\alpha}\right)_{\alpha \in A}$ is uo-Cauchy in $X^{u}$. As $X$ is an ideal in $X^{u}$ and so it is a regular Riesz subspace, it is even enough to show that $\left(x_{\alpha}\right)_{\alpha \in A}$ is uo-Cauchy in $X$ (see [13, Theorem 3.2]). By the first step we know that the net $\left(P_{F} x_{\alpha}\right)_{\alpha \in A}$ is uo-Cauchy, which means that $\left|P_{F} x_{\alpha}-P_{F} x_{\beta}\right| \wedge z \stackrel{o}{\longrightarrow} 0$ for each $z \in X_{+}$. Now for $y$ in $X_{+}$we have

$$
\begin{aligned}
\left|x_{\alpha}-x_{\beta}\right| \wedge y & =P_{F}\left(\left|x_{\alpha}-x_{\beta}\right| \wedge y\right)+P_{F}^{d}\left(\left|x_{\alpha}-x_{\beta}\right| \wedge y\right) \\
& =\left|P_{F} x_{\alpha}-P_{F} x_{\beta}\right| \wedge P_{F} y+P_{\gamma}^{d}\left(\left|x_{\alpha}-x_{\beta}\right| \wedge y\right) \\
& \leq\left|P_{F} x_{\alpha}-P_{F} x_{\beta}\right| \wedge P_{F} y+P_{F}^{d} y .
\end{aligned}
$$

It follows that

$$
\limsup _{(\alpha, \beta)}\left|x_{\alpha}-x_{\beta}\right| \wedge y \leq P_{F}^{d} y \text {. }
$$

As this happens for every $F \in \mathcal{F}$ and $P_{F}^{d} \downarrow 0$, we deduce that $\limsup _{(\alpha, \beta)}\left|x_{\alpha}-x_{\beta}\right| \wedge$ $y=0$, which proves that $\left(x_{\alpha}\right)$ is uo-Cauchy in $X$ as claimed. This completes the proof.

To continue our discussion we need to recall some facts and fix some notations. Recall that a filtration in $X$ is a sequence of conditional expectations $\left(T_{n}\right)_{n \geq 1}$ such that $T_{n} T_{m}=T_{m} T_{n}=T_{n \wedge m}$ for every $n, m \geq 1$. We will assume also that $T_{n} T=T T_{n}=T$ for all $n$. An adapted process is a sequence $\left(x_{n}\right)$ in $X$ such that $x_{n} \in R\left(T_{n}\right)$ for every $n$. An adapted process is called a martingale if $T_{i} x_{j}=x_{i}$ for all $i, j \in\{1,2, \ldots\}$ with $i \leq j$. It is called a submartingale if $T_{i} x_{j} \geq x_{i}$ for all $i, j \in\{1,2, \ldots\}$ with $i \leq j$. We call a stopping time adapted to the filtration $\left(T_{i}\right)_{i \geq 1}$ an increasing sequence $\left(P_{i}\right)_{i \geq 1}$ of band projections on $X$ such that $P_{i} T_{j}=T_{j} P_{i}$ whenever $1 \leq i \leq j$. An example of stopping time can be obtained as above: if $\left(x_{k}\right)$ is an increasing sequence in $X$ with $x_{k} \in R\left(T_{k}\right)^{+}$for $k=1,2, \ldots$ then the sequence $\left(P_{k}=P_{x_{k}}\right)_{k \geq 1}$ is a stopping time. If $P$ is a stopping time, then $Q=P \wedge n$ defined by

$$
Q_{i}=P_{i} \text { if } i<n \text { and } Q_{i}=I \text { if } i \geq n,
$$

is a stopping time. If $\left(x_{i}\right)$ is an adapted process and $P=\left(P_{i}\right)$ is a bounded stopping time, we define the stopped process $\left(x_{P}, T_{P}\right)$ by putting 


$$
x_{P}=\sum_{i=1}^{\infty}\left(P_{i}-P_{i-1}\right) x_{i},
$$

where $P_{0}=0$. We refer the reader to [24, 3] for more information about the subject.

In the next Lemma and Theorem we consider a submartingale $\left(x_{n}, T_{n}\right)_{n \geq 1}$. For each positive real $K$ we consider a sequence of projection bands

$$
B_{K, n}=B\left(x_{1} \leq K e, \ldots, x_{n-1} \leq K e, x_{n}>K e\right) .
$$

We use here the notations adopted by Grobler in his papers (see for example [14]), which are close to the notations used in probability theory. So, $B(x<y)$ denotes the band generated by $(y-x)^{+}$and $B(x \geq y)$ is its disjoint complement. Thus $B_{K, n}$ is the intersection of $n$ bands.

The band $B_{K, \infty}$ is defined as follows:

$$
B_{K, \infty}=B\left(x_{n} \leq K e \text { for all } n\right) .
$$

Let $P_{K, n}$ denote the corresponding band projection for $n \in \mathbb{N} \cup\{\infty\}$. This allows us to define a stopping time $\tau^{K}=\left(\tau_{n}^{K}\right)_{n \geq 1}$ by putting $\tau_{n}^{K}=\sum_{j=1}^{n} P_{K, j}$. If $\left(x_{n}\right)_{n>1}$ is an adapted process then the stopped process $\left(x_{n \wedge \tau^{K}}\right)$ is the process $\left(z_{n}\right)_{n \geq 1}$ given by

$$
z_{n}=\sum_{j=1}^{n-1} P_{K, j} x_{j}+P_{K, n-1}^{d} x_{n}=\sum_{1 \leq j \leq \infty} P_{K, j} x_{j \wedge n} .
$$

Lemma 34 Let $\left(x_{n}, T_{n}\right)_{n \geq 1}$ be a submartingale and $K>0$. If $T\left[\sup _{n}\left(x_{n+1}-\right.\right.$ $\left.\left.x_{n}\right)^{+}\right] \in X^{u}$, then the stopped process $\left(\widetilde{x}_{n}=x_{n \wedge \tau^{K}}\right)$ satisfies

$$
\limsup _{n \longrightarrow \infty} T\left|\widetilde{x}_{n}\right| \in X^{u} .
$$

Proof. We will use the notations preceding Lemma 34 and recalling that

$$
\widetilde{x}_{n}=\sum_{j=1}^{\infty} P_{K, j} x_{j \wedge n}+P_{K, \infty} x_{n}
$$

Now define a new process $\left(y_{n}=x_{n \wedge \tau^{K}-1}\right)$ by putting

$$
y_{n}=\sum_{j=1}^{\infty} P_{K, j} x_{n \wedge j-1}+P_{K, \infty} x_{n}
$$


with $x_{0}=0$ and write

$$
\widetilde{x}_{n}=y_{n}+\widetilde{x}_{n}-y_{n}
$$

We have on one hand,

$$
\widetilde{x}_{n}-y_{n} \leq\left(\widetilde{x}_{n}-y_{n}\right)^{+} \leq V:=\sup _{j}\left(x_{j+1}-x_{j}\right)^{+} .
$$

On the other hand as $P_{B_{K, j}} y_{n}^{+} \leq K P_{B_{K, j}} e$ for all $j \in \mathbb{N} \cup\{\infty\}$ it follows from the definition of $y_{n}$ that

$$
y_{n}^{+} \leq K e .
$$

Moreover, as $\left(\widetilde{x}_{n}\right)$ is a submartingale ([24, Theorem 4.5]) we have

$$
T \widetilde{x}_{n} \geq T \widetilde{x}_{1}=T x_{1} .
$$

As a conclusion, using the above inequalities, we get

$$
\begin{aligned}
T\left|\widetilde{x}_{n}\right| & =2 T \widetilde{x}_{n}^{+}-T \widetilde{x}_{n} \leq 2 T \widetilde{x}_{n}^{+}-T x_{1} \\
& \leq 2 K e+2 T \sup _{n}\left(x_{n+1}-x_{n}\right)^{+}-T x_{1} \in X^{u},
\end{aligned}
$$

which proves the lemma.

The following result generalizes [7, Theorem 5.2.8].

Theorem 35 Let $\left(T_{n}\right)$ be a filtration adapted with $T$ and let $\left(x_{n}, T_{n}\right)$ be a martingale in $X$ satisfying $T \sup \left|x_{n+1}-x_{n}\right| \in X^{u}$. Define the projection band in $X^{u}$ as follows

$$
Q=\sup \left\{P \in \mathfrak{P}: P x_{n} \text { is order convergent in } X^{u}\right\} .
$$

Then $Q^{d}=P_{\left(\sup x_{n}\right)^{\infty}}$.

Proof. Let $x=\sup x_{n}$. By Theorem 33 we know that $\left(Q x_{n}\right)$ is uo-convergent in $X^{u}$. Let $K \geq 1$ be a fixed integer and consider the projection bands $B_{K, n}, n \in \mathbb{N} \cup\{\infty\}$ and denote by $P_{K, n}$ the corresponding band projection and let $\tau^{K}$ be the stopping time defined above. It follows from Lemma 34 that $\limsup _{n \rightarrow \infty} T\left|\widetilde{x}_{n}\right| \in X^{u}$. This implies by [23, Theorem 3.5] that $\left(\widetilde{x}_{n}\right)$ is uo-convergent in $X^{u}$. Thus $\left(P_{K, \infty} \widetilde{x_{n}}\right)$ is uo-convergent in $X^{u}$ as well. But $P_{K, \infty} \widetilde{x}_{n}=P_{K, \infty} x_{n}$ and then $P_{K, \infty} x_{n}$ is uo-convergent in $X^{u}$. It follows from 
Theorem 33 that $\left(P x_{n}\right)$ is uo-convergent in $X^{u}$ where $P=\sup _{K} P_{K, \infty}$. To prove the theorem it is enough to observe that

$$
\bigvee_{k=1}^{\infty} B_{K, \infty}=\bigvee_{k=1}^{\infty} B_{(x-K e)^{+}}^{d}=B_{\left(\sup x_{n}\right)^{\infty}}^{d}
$$

Or, equivalently,

$$
\bigwedge_{k=1}^{n} B_{(x-k e)^{+}}=B_{x^{\infty}}
$$

But this is exactly what Theorem 19 says.

As a corollary to this theorem we can observe that in the statement of Theorem 5.28 in [7], $\mathbb{P}\left(\lim \inf x_{n}=-\infty\right)=0$. Furthermore the set $A_{2}$ can be defined as $A_{2}=\left\{\sup X_{n}=\infty\right\}$ instead of $\left\{\lim \sup X_{n}=\infty\right\}$.

The following generalizes Lévy's Theorem to the frame of Riesz spaces (see [8, Theorem 5.1.2]).

Theorem 36 Let $\left(T_{n}\right)$ be a filtration adapted with $T$ and let $\left(P_{n}\right)$ be a sequence of band projections satisfying $P_{n} T_{n}=T_{n} P_{n}$ for each integer $n$. Then the series $\sum_{n=1}^{\infty} P_{n}$ e and $\sum_{n=1}^{\infty} T_{n-1} P_{n}$ e have the same infinite part.

Proof. Define for each $n, d_{n}=P_{n} e-T_{n-1} P_{n} e$ and $x_{n}=d_{1}+\ldots+d_{n}$. Then $\left(x_{n}\right)_{n \geq 1}$ is a martingale and $\left|x_{n+1}-x_{n}\right|=\left|d_{n+1}\right| \leq e$. Then Theorem 35 can be applied. It is sufficient to show that $Q_{i} \sum_{n=1}^{\infty} P_{n} e$ and $Q_{i} \sum_{n=1}^{\infty} T_{n-1} P_{n} e$ have the same infinite part for $i=1,2$, where $Q_{1}=Q$ and $Q_{2}=I-Q$. But this is trivial for $Q_{1}$ because the sequence

$$
Q_{1} x_{n}=Q_{1}\left[\sum_{k=1}^{n} P_{k} e-\sum_{k=1}^{n} T_{k-1} P_{k} e\right]
$$

is order convergent in $X^{u}$, and it is also true for $Q_{2}$ as an immediate consequence of the following inequalities:

$$
x_{n} \leq \sum_{k=1}^{n} P_{k} e \quad \text { and } \quad-x_{n} \leq \sum_{k=1}^{n} T_{k-1} P_{k} e, \quad n \geq 1 .
$$

The result now follows from Proposition 21. 


\section{References}

[1] Y. Azouzi, Completeness for vector lattices, J. Math. Anal. Appl.472 (2019) 216-230.

[2] Y. Azouzi; F. Ben Amor, Subspaces and operators with the Stone condition, Positivity, 14, (2010), 585-593.

[3] Y. Azouzi and K. Ramdane, Burkholder Inequalities in Riesz spaces, Indagationes Mathematicaes, Indag. Math. (N.S.) 28 (2017), no. 5, 1076-1094.

[4] Y. Azouzi, W.C. Kuo, K. Ramdan, B.A. Watson, Convergence in Riesz spaces with conditional expectation operators, Positivity (2015) 19:647657

[5] Y. Azouzi and K. Ramdane, Burkholder theorem in Riesz spaces, preprint.

[6] Y. Azouzi, M. Trabelsi, Lp-spaces with respect to conditional expectation on Riesz spaces, J. Math. Anal. Appl., 447(2017), 798-816.

[7] L. Breiman, Probability, Addision Wesley, California, 1968.

[8] T. K. Chandra, The Borel-Cantelli Lemma, Springer Briefs in Statistics. Heidelberg: Springer, 2012.

[9] Tapas Kumar Chandra \& Andrew Rosalsky, A curious application of the Borel-Cantelli Lemmas, a result of Barndorff-Nielsen, and some open problems, Stoch. Anal. Appl. 37 (2019), no. 3, 473-479.

[10] Y. Deng, M O'BrienV.G. Troitsky, Unbounded norm convergence in Banach lattices, Positivity 21 (3) (2017) 963-974.

[11] K. Donner, Extension of Positive Operators and Korovkin Theorems, Springer (1982).

[12] R. Durrett, Probability Theory and exemples, Fourth Edition

[13] N. Gao, V. Troitsky, F. Xanthos, Uo-convergence and its applications to Cesàro means in Banach lattices, Israel J. Math. 220 (2017) 649-689 
[14] J.J. Grobler, Jensen's and martingale inequalities in Riesz spaces, Indag. Math. 25 (2014) 275-295.

[15] J.J. Grobler, Stopped processes and Doob's optional sampling theorem, J. Math. Anal. Appl. 497 (2021), no. 1, 124875.

[16] J.J. Grobler, The Kolmogorov-Č́ntsov theorem and Brownian motion in vector lattices. J. Math. Anal. Appl. 410, 891-901 (2014)

[17] J.J. Grobler, C.C.A. Labuschagne, The Itô integral for Brownian motion in vector lattices: Part 1., J. Math. Anal. Appl., 423, 797-819 (2015).

[18] J. J. Grobler, C.C.A. Labuschagne, The Itô integral for Brownian motion in vector lattices: Part 2., J. Math. Anal. Appl., 423, 820-833 (2015)

[19] M. Kandić, H. Li, Troitsky, Unbounded norm topology beyond normed lattices, Positivity (2018) 22:745-760

[20] M. Kandić, M. Marabeh, V.G. Troitsky, Unbounded norm topology in Banach lattices, J. Math. Anal. Appl. 451 (1) (2017) 259-279.

[21] Kaplan, On unbounded order cv, Real Anal. Exchange 23 (1) (19981999) 75-184.

[22] W. C. Kuo, C. A. Labuschagne, and B. A. Watson, Conditional expectations on vector lattices, J. Math. Anal. Appl., 303 (2005), 509-521.

[23] W.-C. Kuo, C.C.A. Labuschagne, B.A. Watson, Convergence of Riesz space martingales, Indag. Math., 17 (2006), 271-283.

[24] W.C. Kuo, C.A. Labuschagne, B.A. Watson, Discrete-time stochastic processes on Riesz spaces, Indag. Math. 15 (2004) 435-451.

[25] W.C. Kuo, C.C.A. Labuschagne, B.A. Watson, Zero-one law for Riesz space and fuzzy process, Zero-one laws for Riesz space and fuzzy random variables. In: Liu, Y., Chen, G., Ying, M. (eds.) International fuzzy systems association. Tsinghua University Press, Springer, cop., Beijing (2005)

[26] Hui Li, Zili Chen,: Some loose ends on unbounded order convergence, Positivity 22(1), 83-90 (2018) 
[27] Myasha Mushambi, Bruce A. Watson, Bertin Zinsou, Generalization of the theorems of Barndorff-Nielsen and Balakrishnan-Stepanov to Riesz spaces. Positivity 24 (2020), no. 3, 753-760.

[28] M. O'Brien, V.G. Troitsky, and J.H. van der Walt, Net convergence structures with applications to Vector lattices, arXiv:2103.01339 [math.FA] 